\title{
Some Observations of Flow Patterns and Statistical Properties of Three Component Flows
}

\section{H. K. Kytömaa}

\section{E. Brennen}

Department of Mechanical Engineering, California Institute of Technology, Pasadena, Calif. 91.125
Vertical air-water flows, solids-water flows and three component air-solids-water flows were investigated in a Three Component Flow Facility. Visual observations of the flow patterns show that three component flows undergo transition and can exhibit strong unsteady vortical motions. Measurements of the fluctuations in crosssectionally averaged volume fraction measurements were made. The statistical properties of the fluctuations are presented in terms of their amplitude and coherent time scale in the form of the Signal To Noise Ratio (STNR) and the Time Constant $(\xi)$, respectively. Remarkably, the solids-water flows and the dispersed bubbly airwater flows exhibit almost identical values of STNR for the same volume fraction. Equally remarkable in the linear relationship between the Time Constant and the mean bubble or particle velocity; this relationship is found to have the same constant of proportionality for both species in the well behaved disperse regime. In the twocomponent churn-turbulent and the three-component agitated vortical regimes, the variables $\xi$ and STNR significantly deviate from their dispersed regime values. The onset of large coherent structures characteristic of these regimes is reflected by a rise in the amplitude of the fluctuations and a marked increase in their coherent time scale. The results of this study demonstrate the large information content in the fluctuations of the measured quantity, both as a flow regime indicator and as a measure of flow quantities in two- and three-component flows.

\section{Introduction}

The discrete nature of multi-component flows is well known to be the cause of fluctuations in measured flow quantities. The statistics of these fluctuations depend on the structure and spatial distribution of the discrete phase. A number of investigators have indeed demonstrated direct relationships between flow properties and the statistics in the measured "noise" that have allowed the extraction of information from the observed fluctuations. For example, the fluctuating component of the pressure drop in a two-phase flow through an orifice was used to derive the flow rates of the components by Ishagai et al. (1965). Jones and Zuber (1975) used the probability density function of X-ray attenuation volume fraction signals as a flow pattern discriminator for vertical bubbly, slug and annular flows. Bernier (1981) used the inherent noise of resistive volume fraction signals in his experimental analysis of kinematic wave propagation in bubbly flows. The significance of the statisical properties of volume fraction measurements depends entirely on the size of the influence volume of the measuring device. For example, hot wire anemometers, optical probes and other devices have been employed to make

\footnotetext{
${ }^{1}$ Present address: Department of Mechanical Engineering, Massachusetts Institute of Technology, Cambridge, Mass. 02139.

Contributed by the Fluids Engineering Division of the THE AMERICan SociETY OF MECHANICAL ENGINEERS and presented at the International Symposium on Slurry Flows, ASME Winter Annual Meeting, Anaheim, Calif., December 7-12, 1987. Manuscript received by the Fluids Engineering Division June 13, 1986; revised manuscript received March 25, 1987.
}

point measurements of volume fraction. To obtain useful in formation, time averaging of the output is necessary. This limits the dynamic resolution of such a device. On the other hand, $\mathrm{X}$-ray and Gamma-ray attenuation techniques inherently carry out line averages along the beam. Capacitive and resistive measuring techniques yield a volume average of concentration. The size and shape of the measuring volume is determined by the geometry of the electrodes. With a large averaging volume, good dynamic response is achieved at the cost of diminished spatial resolution.

In this study we exploit the fluctuation in the measured volume fraction signal to investigate the structure of two- and three-component flows as they undergo transition from a uniform to an agitated regime. We demonstrate that the fluctuations in the signal can be used to measure flow quantities. The flows considered consist of air bubbles (average diameter of $4 \mathrm{~mm}$ ) and polyester particles (average diameter $3 \mathrm{~mm}$ ) in a continuous medium of water. Real time volume fraction measurements were made using an Impedance Volume Fraction Meter (IVFM) (Bernier (1981), Kytömaa (1986)).

The fluctuations in the volume fraction signal are characterized by two properties of their auto-correlation function; its amplitude and "width"' (coherent time scale). These two quantities are easily measured and are useful characterizations of the flow structure. The visual observations of the transition of two- and three-component flows are related to these statistics. The results suggest a non-intrusive method of 
measuring disperse flow quantities, namely the total volume fraction and velocity, for two- and three-component flows.

In the work presented here, Reynolds numbers of 1000 and 400 were measured for individual bubbles and particles respectively based on relative velocity. These values are significantly higher than the Reynolds numbers in other three-component flow studies by Fessas and Weiland (1981) and Batchelor (1986). Flow regime transitions were nevertheless observed in all these studies.

\section{Experimental Facility and Instrumentation}

The Three Component Flow Facility (TCFF) shown in Fig. 1 was used to study the statistical properties of volume fraction signals in bubbly, slurry and three component flows. The test section is a vertical clear acrylic pipe .1016 meters (4 in.) in diameter and 2.2 meters in length. The air-water flows are formed by introducing the gas through an injector situated inside the vertical pipe, .5 meters below the test section. The injector consists of an array of twelve $3.2 \mathrm{~mm}$ (1/8 in.) diameter brass tubes perforated with $.4 \mathrm{~mm}$ (1/64 in.) holes. An $8 \mathrm{~atm}$ (120 psi) compressed air line supplies the injector through a regulator, an orifice plate flow meter (to monitor air mass flow), valves to control air flow and a manifold to distribute the air flow evenly among the brass tubes. The slurry flows studied consist of water and polyester particles. The polyester particles are of the type used for molding applications (specific gravity $=1.43$ ). They are smooth and have a cylindrical shape with an equal mean height and diameter of $3 \mathrm{~mm}( \pm .5 \mathrm{~mm})$. The most novel aspect of the facility is its ability to handle solids and to control their flow rate independently of the liquid without having to add or remove solids from the system. When at rest prior to an experiment the solids are trapped between a vertical 4 inch control cylinder and the storage hopper (see Fig. 1). As the control cylinder is raised from the reducer on top of which it sits, the gap created allows particles to enter the test section under the action of gravity. The vertical position of the control cylinder can be varied by means of a control rod attached to a worm gear mechanism and this permits the solids flow rate to be controlled by varying the gap between the cylinder and the reducer. To recycle the solids after an experiment the control cylinder is lowered to the closed position and sufficient upward water flow is generated to fluidize the solids in the lower tank and to carry them back to the upper tank where they settle into their original position.

The static pressure gradient in the test section is monitored using an inductive differential pressure transducer. The volume fraction of the dispersed medium is measured using an Impedance Volume Fraction Meter (IVFM). The IVFM was

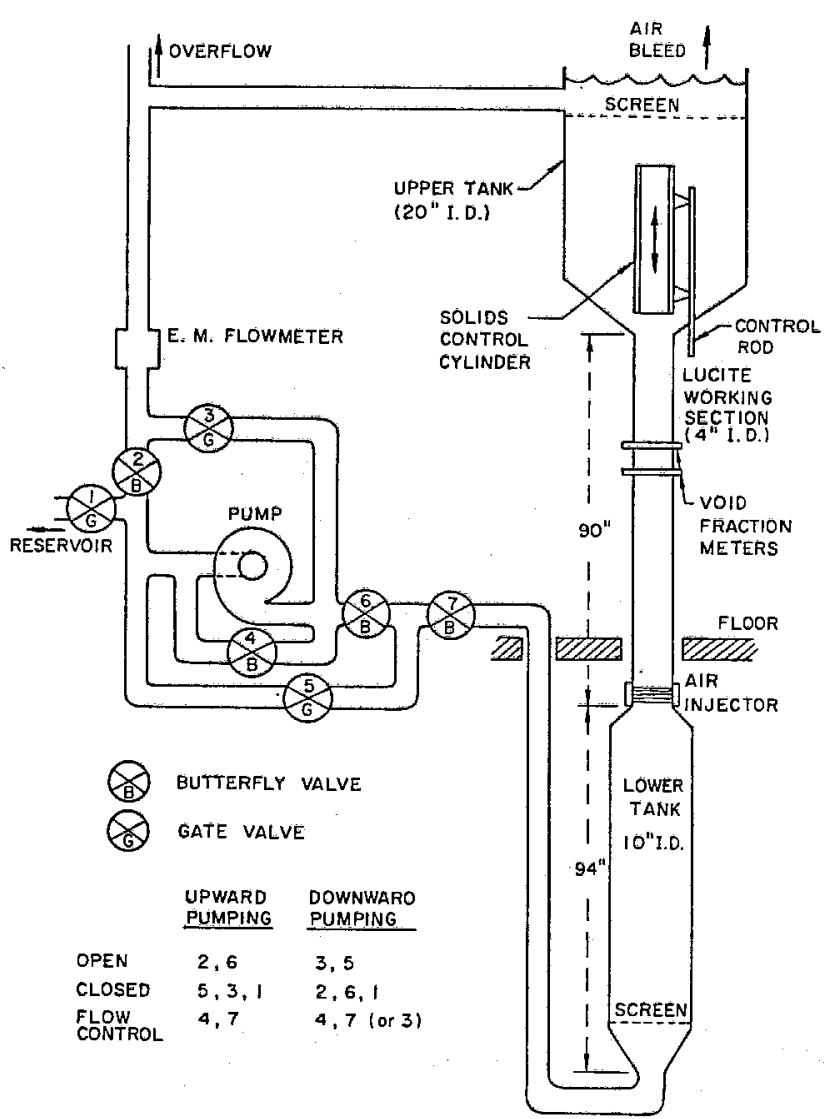

Fig. 1 Schematic of the Three Component Flow Facility

developed by Bernier (1981). It has been modified for temperature compensation and now has a shielded electrode configuration which decreases the axial extent of the influence volume over which the measurement is carried out. The active stainless steel electrodes which are flush mounted into a section of .1016 meter (4 in.) diameter non-conducting acrylic pipe are $6.4 \mathrm{~mm}$ in axial length and form diametrically opposed 90 degree arcs on the circumference of the pipe. The active electrodes are each sandwiched between two shielding electrodes. These are $9.5 \mathrm{~mm}$ in axial length and also form 90 degree arcs. Figure 2 shows the electrode configuration. The shielding electrodes duplicate the active electrode potential through a high input impedance voltage follower. The IVFM is excited at an amplitude of .3 volts r.m.s. and a frequency of

\section{Nomenclature}

ACF = Auto-Correlation Function of the random fluctuations in the volume fraction signal, $\left(\mathrm{V}^{2}\right)$

$j=$ total volume flux, $(\mathrm{m} / \mathrm{s})$

$j_{g}=$ gas volume flux, $(\mathrm{m} / \mathrm{s})$

$j_{p}=$ solids volume flux, $(\mathrm{m} / \mathrm{s})$

$j_{p j}=$ solids drift flux, $(\mathrm{m} / \mathrm{s})$

$\stackrel{L}{L}=$ vertical separation between pressure taps, (m)

$\Delta p=$ hydrostatic pressure difference in a threecomponent mixture between the pressure taps, relative to that in water, $\left(\mathrm{N} / \mathrm{m}^{2}\right)$

$$
\begin{aligned}
R_{\tilde{V} \tilde{V}}= & \begin{array}{l}
\text { Auto-correlation Function } \\
\text { of the random, fluctua- } \\
\text { tions in the volume frac- } \\
\text { tional signal, }\left(\mathrm{V}^{2}\right)
\end{array} \\
\text { STNR }= & \begin{array}{l}
\text { signal to noise ratio of the } \\
\text { volume fraction signal, } \\
\end{array} \\
& (\mathrm{m} / \mathrm{s}) \\
v_{g}= & \text { mean gas velocity, }(\mathrm{m} / \mathrm{s}) \\
v_{l}= & \text { mean liquid velocity, }(\mathrm{m} / \mathrm{s}) \\
v_{p}= & \text { mean solids velocity, }(\mathrm{m} / \mathrm{s}) \\
v_{g(p l)=}= & \text { mean gas velocity relative } \\
& \text { to the combined solids and } \\
& \text { liquid volume flux, }(\mathrm{m} / \mathrm{s}) \\
\bar{V}= & \text { mean value of the volume } \\
& \text { fraction signal, }(\mathrm{V})
\end{aligned}
$$

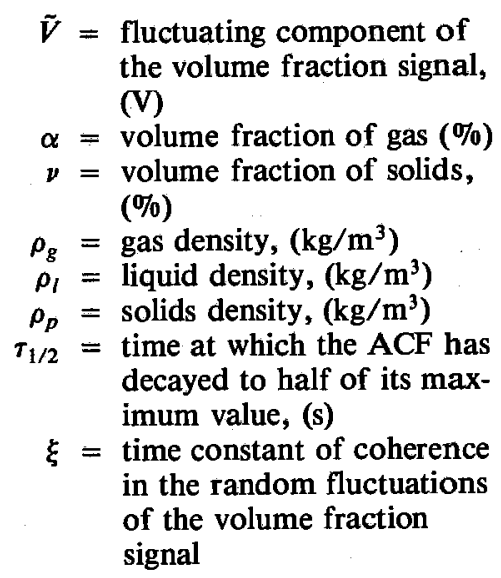




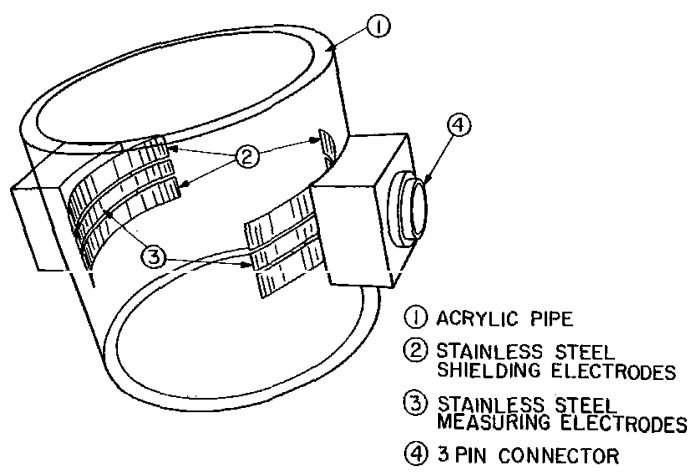

Fig. 2 Isometric view of the shielded Impedance Volume Fraction Meter electrode geometry

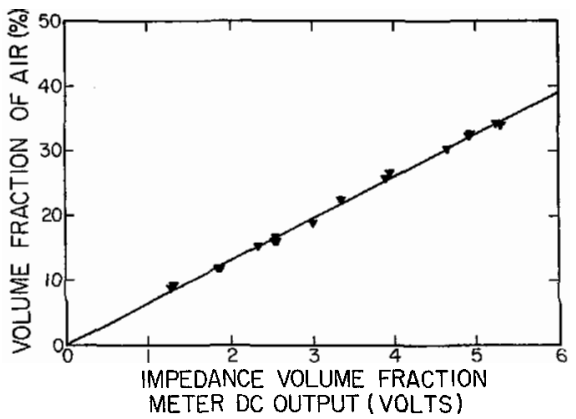

Fig. 3 Steady state calibration of the Impedance Volume Fraction Meter with bubbly flows for zero water volumetric flux. The volume fraction was derived from the hydrostatic pressure gradient measurement.

$40 \mathrm{KHz}$ at which the impedance is found to be primarily resistive. The excitation and signal processing equipment is described in more detail by Bernier (1981). The IVFM is calibrated with both bubbly and particulate flows against the volume fraction ( $\alpha$ and $\nu$, respectively) obtained from the static pressure gradient measurement. These two plots are shown in Figs. 3 and 4. Equations (1) and (2) are the respective linear regression fits of the shown plots. The corresponding correlation coefficients for these fits are .9992 and .997 . The discrepancy between the two fits (up to 4 percent) is attributed to experimental error in the measurement.

$$
\begin{gathered}
\alpha(\%)=6.53 \text { IVFM(Volts) }-.006 \\
\nu(\%)=6.77 \text { IVFM(Volts) }-.41
\end{gathered}
$$

The IVFM is found to have excellent linearity up to the volume fractions of at least $40 \%$. With a sensitivity of .15 Volts per percent of volume fraction, and the passage of individual bubbles (or particles) is readily detectable.

\section{Experimental Procedure and Presentation of Results}

After initiation of each experiment, data were not taken for 30 seconds in order to permit passage of the initial transient. Then observations of the flow pattern were made. For each run, measurements were made of the air flow rate (using the orifice meter), the liquid flow rate (measured with an electromagnetic flow meter). The total flow rate was constrained by flooding of either the solid or the gas phase and the total flux was restricted to the range of $-.1 \mathrm{~m} / \mathrm{s}$ to $.2 \mathrm{~m} / \mathrm{s}$. The IVFM d.c. output and the static pressure transducer output were monitored on a strip chart recorder. In two component flows, $\nu$, the solids volume fraction or $\alpha$, the air volume fraction is directly obtained from the IVFM. At low flow rates $\alpha$ or $v$ is also given by the output of the differential pressure transducer since the frictional component of the pressure drop is very small. Indeed this is how the calibration equations (1) and (2) were obtained. In a three-component flow at low flow

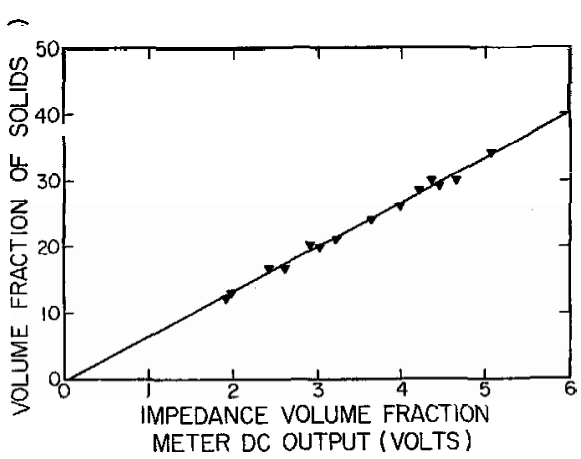

Fig. 4 Steady state calibration of the Impedance Volume Fraction Meter with particulate flows for zero total zero total volumetric flux. The volume fraction was derived from the hydrostatic pressure gradient measurement.

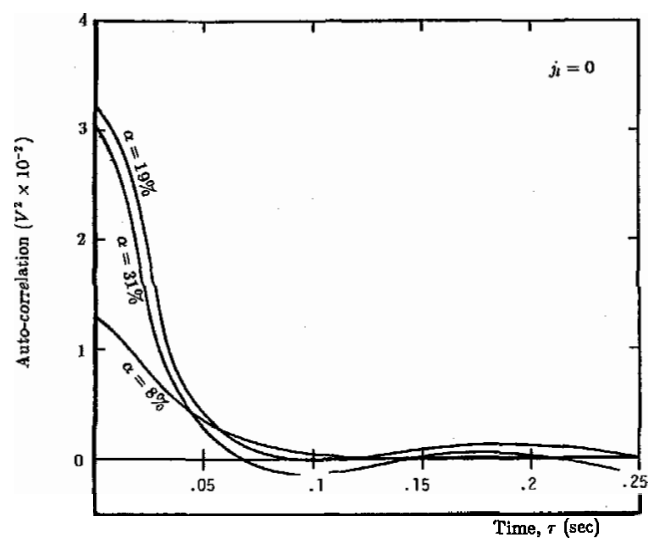

Fig. 5 Typical auto-correlograms of the IVFM output fluctuations in a steady bubbly flow regime obtained from the HP 3562 Digital Signal Pro. cessor for a 60 second record

rates, the differential static pressure transducer yields the bulk density which is a function of the individual volume fractions of air and solids. The mean IVFM d.c. output gives the sum of the air and solid volume fractions. Thus we can deduce the concentration of the individual constituents.

The IVFM a.c. component was recorded on magnetic tape through a d.c. blocking amplifier with a $3 \mathrm{~dB}$ cut off frequency of $.032 \mathrm{~Hz}$ and a fall off slope at $10 \mathrm{~dB}$ per octave. The record length was five minutes whenever possible. The shortest record was of one minute which proved adequate for accurate determination of IVFM output fluctuation statistics.

The size distribution of a multicomponent flowing medium influences the statistical properties of the fluctuating component of volume fraction signals. For example, for two flows of equal volume fraction, the one with large particles will yiẹld less frequent and larger fluctuations in the measurement than the one with very small particles. In this paper we used the properties of the Auto-Correlation Function (ACF) of the IVFM output fluctuations to characterize the flow. Typical ACF records are shown in Fig. 5. The two dominant features of the AFC are a) its amplitude $\left(R_{\tilde{L} \tilde{V}}(0)\right)$ which is the mean square magnitude of the fluctuations, and $b$ ) the time at which the function has decayed to a certain fraction of its peak value. The latter is interpreted as a measure of the coherent time scale. These two quantities were used to characterize the structure of a variety of two- and three-component flows. The amplitude $\left(R_{\tilde{V} \tilde{V}}(0)\right)$ is presented in terms of the dimensionless Signal To Noise Ratio (STNR), and the coherent time scale is presented in terms of the time constant $\xi$; these variables are defined below:

$$
\operatorname{STNR}=\frac{\bar{V}}{\sqrt{R_{\bar{\nu} \tilde{V}}(0)}},
$$




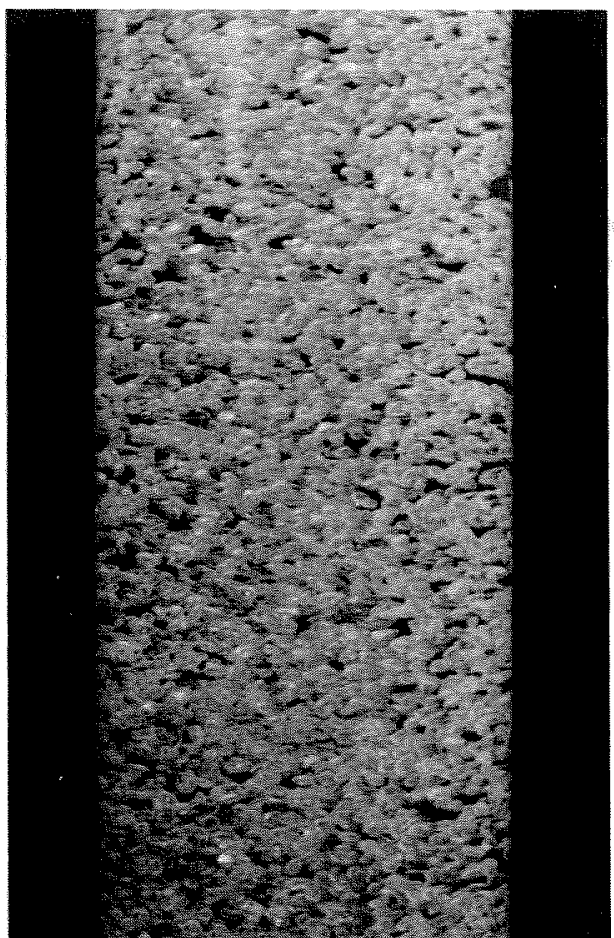

Fig. 6 Bubbly-air water flow of 8 percent volume fraction

where $\bar{V}$ is the mean d.c. IVFM output voltage, and

$$
\xi=1.6783 / \tau_{1 / 2} \text {, }
$$

where $\tau_{1 / 2}$ is the time at which the ACF has decayed to half of its maximum value. A Hewlett Packard 3562 spectral analyser was used to obtain the Auto Correlation Functions (ACF) of IVFM output fluctuations. A chosen ensemble length of one second proved to be adequate to give a repeatable ensemble averaged ACF records. Simultaneous visual observations were made of the nature of the flows and the flow pattern. Measurements of time constants $\xi$ and the STNR are related below to the disperse medium volume fraction and mean velocity, and observations of the flows.

\section{Air-Water Flows}

The air-water flows are created by injecting air bubbles into water. At low air volume fractions, the resulting twocomponent medium consists of a homogeneous distribution of bubbles with a photographically measured average bubble size of $4 \mathrm{~mm}$ and a deviation of up to $.5 \mathrm{~mm}$ from the mean. The flow remains uniform up to a volume fraction of approximately 42 percent. This type of flow is known as the dispersed bubbly flow, an example of which is shown in Fig. 6. As the flow rate of the injected air is increased, the two-component medium becomes intermittently agitated and the formation of large bubbles becomes visible. This flow is said to be churnturbulent. A photograph of such a flow (Fig. 7) shows the presence of large bubbles. Transition from bubbly flow to churn-turbulent is observed to happen more suddenly than the change in the opposite direction. An initially churn-turbulent flow takes of the order of minutes to settle down to a bubbly state when the air of the initially agitated medium is decreased. The change in regime has the notable effect of preventing the volume fraction from ever rising above $\mathbf{4 5}$.

The structure of these air-water flows is characterized using the statistics of the fluctuations of measured volume fraction signals. Two statistical criteria are used, namely the Signal To Noise Ratio (STNR) which is the ratio of the d.c. IVFM output and the amplitude of the fluctuations, and the time constant, $\xi$, which is inversely proportional to the coherent time

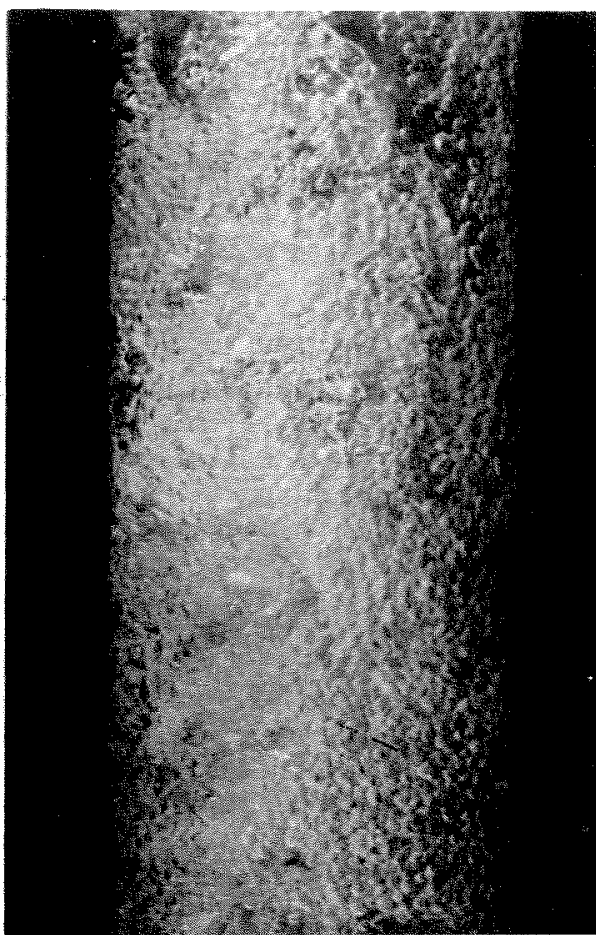

Fig. 7 Churn-turbulent air-water flow of 37 percent volume fraction

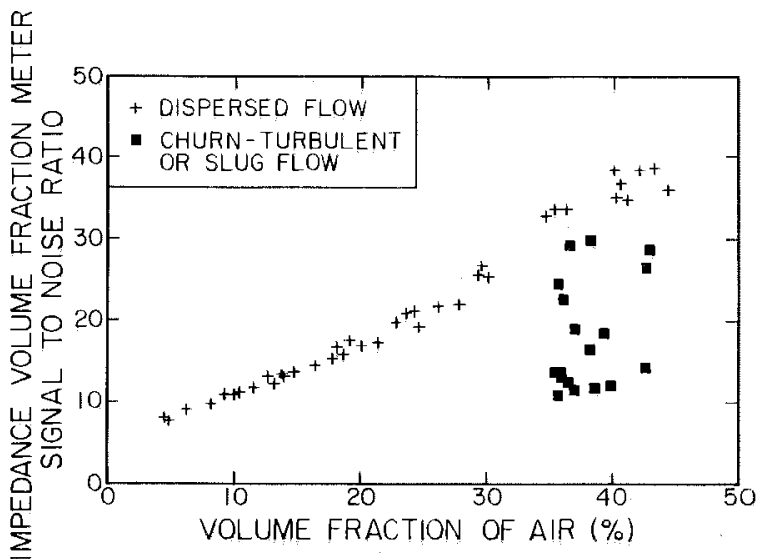

Fig. 8 Signal To Noise ratio of the Impedance Volume Fraction Meter output for bubbly and churn-turbulent flows

scale of the "noise." These variables were derived from experimentally obtained auto-correlation records of the fluctuating signal.

The Signal To Noise Ratio (STNR) of the IVFM was found to behave in two distinct ways depending on whether the flow is bubbly or churn-turbulent. In bubbly air-water flows, the STNR rises monotonically as the volume fraction is increased up to 45 percent. Upon additional air injection, a sudden drop in STNR is experienced along with a slight decrease in volume fraction as shown in Fig. 8. The drop in STNR coincides with the formation of slugs of air present in churn-turbulent flows. The accompanying drop in void fraction is caused by the increase in the mean relative velocity and the perservation of continuity at the air flow rate at which transition occurs. Thus there are two stable values of signal to noise ratio for volume fractions between 35 and 45 percent.

The time constant, $\xi$, in bubbly flows is found to be linearly proportional to the mean bubble velocity, $v_{g}$. This quantity is derived from the measured air flux $j_{g}$ and its volume fraction, $\alpha$ using the following equation: 


$$
v_{g}=\frac{j_{g}}{\alpha}
$$

The results for the time constant, $\xi$, are shown in Fig. 9 plotted against the mean air velocity. The bubbly flow results are indicated by the encircled points. These all fall on a straight line (slope $\approx 370 \mathrm{~m}^{-1}$ and zero vertical axis intercept). In other words, the coherent time scale of fluctuations caused by the passage of individual bubbles past the IVFM electrodes is found to be linearly proportional to their average residence time in the field of influence of the device (the electric field caused by the electrodes). However, at onset of churnturbulence, the magnitude of the time constant decreases sharply by an order of magnitude. This decrease is indicative of the formation of large structures in the agitated flow regime. Constant liquid flux curves assume a ' $C$ '' shape; four such curves are presented. For a constant liquid flux, the lower volume fraction flows exhibit higher values of $\xi$ since bubble interactions cause their terminal velocity to decrease with volume fraction. The marked decrease in $\xi$ caused by the onset of churn-turbulence occurs as the air volume fraction is increased above its critical value of approximately 42 percent. Points on the vertical part of the constant liquid flux curves are considered to be transitional, and points on the rising lower branch of these curves are considered "fully developed." Churn-turbulent data are presented on a separate

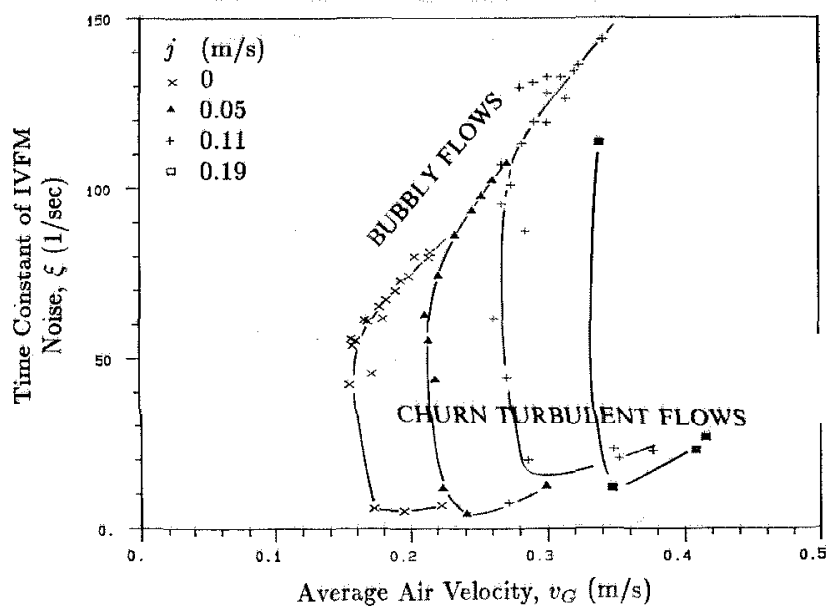

Fig. 9 Time constani $\xi$ (reciprocal of the coherent time scale) of the fluctuating IVFM output slgnal in bubbly and churn-turbulent flows versus the average air velocity. Note the dramatic drop in $\xi$ on onset of churn-turbulence.

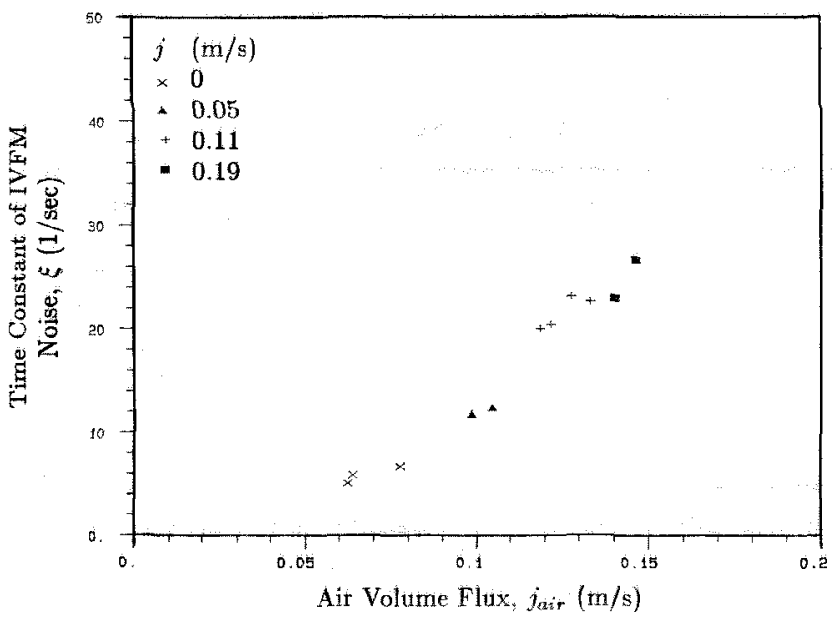

Fig. 10 Time constant $\xi$ of the fluctuating IVFM signal in fully developed chum-turbulent flows versus the air volumetric flux, showing all points on the one curve with little scatter graph. Values of $\xi$ for fully developed churn-turbulent flows

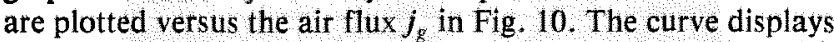
a monotonic increase of the time constant with air flux with less scatter than the corresponding points in Fig. 9 .

These findings demonstrate the rich information content in the noise of the measured volume fraction signal. The STNR is a dimensionless quantity, and it is independent of the sensitivity (gain) of the measuring device (in our case the IVFM). The consistent relationship between the STNR and volume fraction for bubbly flows indicates a possible method of monitoring volume fraction using the STNR in situations where the

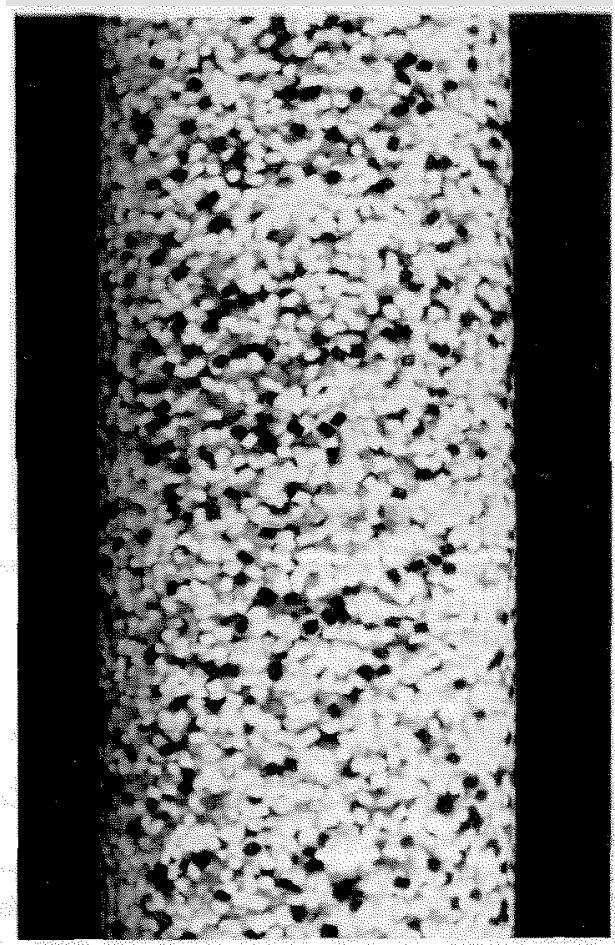

Fig. 11 Dispersed solids water flow of 12 percent volume fraction

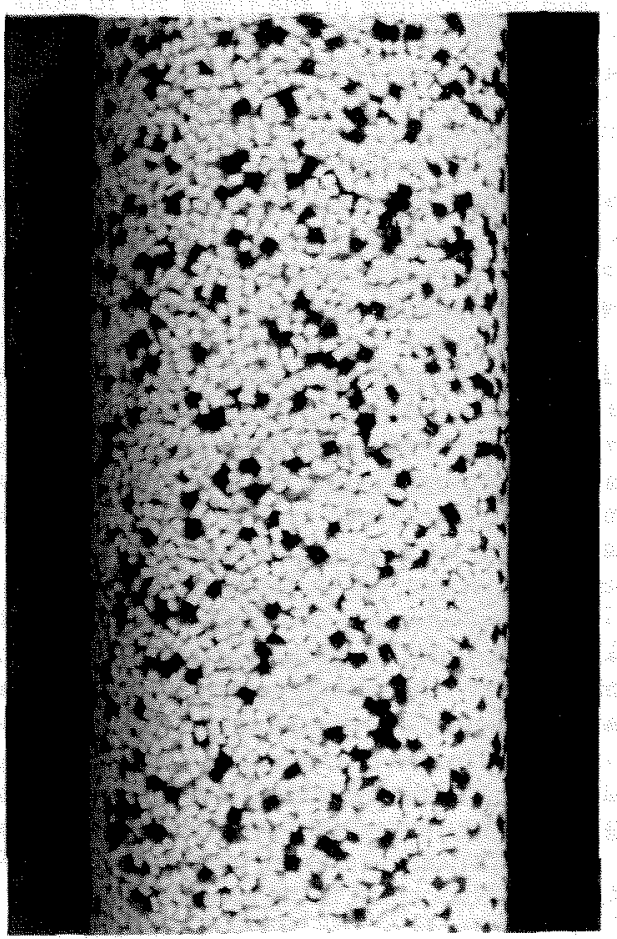

Fig. 12 Solids-water plug flow of 55 percent volume fraction 
recalibration of the monitoring device is not convenient or is impossible. The STNR, used in conjunction with the volume fraction provides a flow regime delimiting tool capable of differentiating between bubbly and churn-turbulent flows. The obtained values of the time constant, $\xi$, suggest that it could be used to determine the mean air velocity and flux of both bubbly and churn-turbulent flows in a non-intrusive fashion. The magnitude of $\xi$ is also found to be sensitive to the flow regime and thus a good indicator of it.

\section{Solid-Liquid Flows}

The solid-liquid flows studied consist of polyester particles of an average diameter of $3 \mathrm{~mm}$. These flows do not display any distinct change in flow regime and remain disperse for the range of total volume flux considered $(0-.24 \mathrm{~m} / \mathrm{s})$ and volume fractions of up to 60 percent. Figure 11 shows a disperse solids-water flow of 12 percent volume fraction. At large volume fractions, the particles are no longer able to move relative to one another and they appear to translate as a solid plug inside the pipe. Such a plug flow is shown in Fig. 12.

We take advantage of the absence of instability in solidswater flows to investigate whether the useful results for disperse bubbly flows hold for particulate flows. Namely that the STNR can be used as a measure of total volume fraction and that the time constant $\xi$ is linearly proportional to the average disperse medium velocity.

Particle flow STNR values are shown versus the volume fraction of solids in Fig. 13. All STNR values fall on a single monotonically increasing curve. Remarkably, this curve coincides with the bubbly flow curve (deviations are within the present experimental scatter), despite the difference in diameter between the particles and bubbles. Measured values of $\xi$ are presented versus volume fraction for different total fluxes in Fig. 14. In the solid-liquid flows, $\xi$ behaves linearly with respect to the particle velocity $v_{p}$. It gradually decreases with $\nu$ for constant total flux (the settling velocity decreases with increased volume fraction) and for a given value of $\nu$, the higher the total flux the larger the resulting $\xi$.

In the present experimental facility, we have no direct means of evaluating the particle flow rate or speed. An indirect method was devised for this purpose. We determined the particle Drift Flux Curve from kinematic shock speed measurements, and then used this result to determine the particle velocity for comparison. The estimate of the solids average velocity is given by

$$
v_{p}=\frac{j_{p j}}{\nu}+j
$$

which is derived directly from the definition of the drift flux.

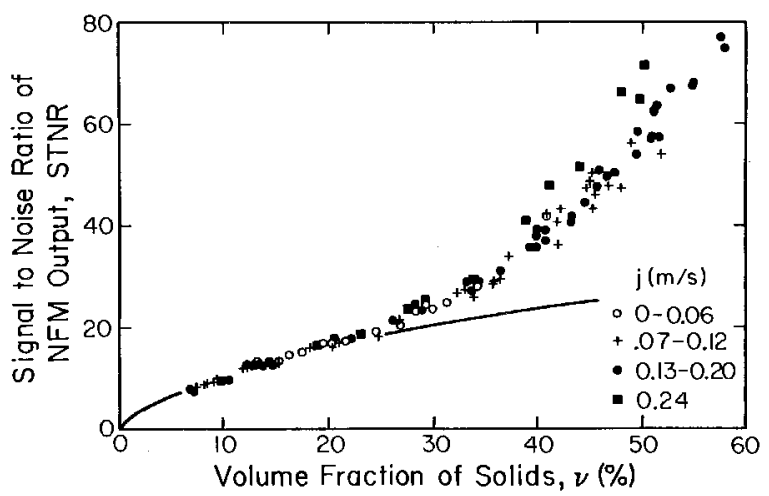

Fig. 13 Signal To Noise ratio of IVFM output for particle flows of different total fluxes plotted against the volume fraction of solids. Also shown is the theoretical hali power curve. The results deviate from this at an approximate volume fraction of 25 percent.
The velocity given by equation (6) is not an exact measure since $j_{p j}(v)$ was derived for zero total flux, and it is now applied to non-zero flowrates. However, for the maximum total flux considered $\left(.25 \mathrm{~m} / \mathrm{s}\right.$ which corresponds to $\left.\operatorname{Re}=25 \times 10^{3}\right)$ the error in the disperse medium velocity is small. Experiments to measure $\xi$ were carried out for volume fractions ranging from 6 to 55 percent. The results, presented in Fig. 14, show that the time constant, $\xi$, is indeed linearly proportional to the mean particle velocity, and the constant of proportionality is almost exactly the same as for bubbly flows. Therefore the suggested method based on the determination of the characteristic coherent time scale of the IFVM "noise" can truly be used as a non-intrusive measurement of the disperse medium velocity with the maximum error of \pm 25 percent of the measurement for the lower velocities considered.

\section{Gas-Solid-Liquid Three-Component Flows}

Three component flows consisting of polyester particles ( 3 $\mathrm{mm}$ diameter) and air bubbles (uncoalesced diameter of $4 \mathrm{~mm}$ ) in a continuous medium of water were investigated for low total fluxes $(j<.06 \mathrm{~m} / \mathrm{s})$, and total volume fractions up to 50 percent.

At the flow rates considered, the pressure gradient in the vertical test section is predominantly hydrostatic. Thus, neglecting the wall effect at these low flow rates, the pressure gradient is linearly proportional to the bulk density which is a function of the volume of the individual constituents. We measure the pressure gradient in the medium using a differential pressure transducer between two taps located a distance $L=1.694 \mathrm{~m}$ apart. The lines leading to the device are filled with water; therefore the measurement is relative to the hydrostatic pressure difference in water over the same height. Hence, the device output pressure can be presented as a linear combination of the disperse medium volume fractions:

$$
\Delta p=\rho_{l} L\left[\nu\left(\rho_{p}-\rho_{l}\right)-\alpha\left(\rho_{l}-\rho_{g}\right)\right] .
$$

where $\nu$ is the volume fraction of solids and $\alpha$ the volume fraction of air. The mean IVFM output gives the sum of the air and solid volume fractions. Since the material constants in (7) are known, the volume fractions can therefore be computed from the two measurements.

6.1 Three-Component Flow Pattern Observations. One of the distinctive characteristics of the three component flows is the dramatic increase in the level of audible noise caused by particle impacts against one another and against the pipe wall. This was found to be true for all volume fractions of air and solids. It is interpreted as a consequence of the increase in the

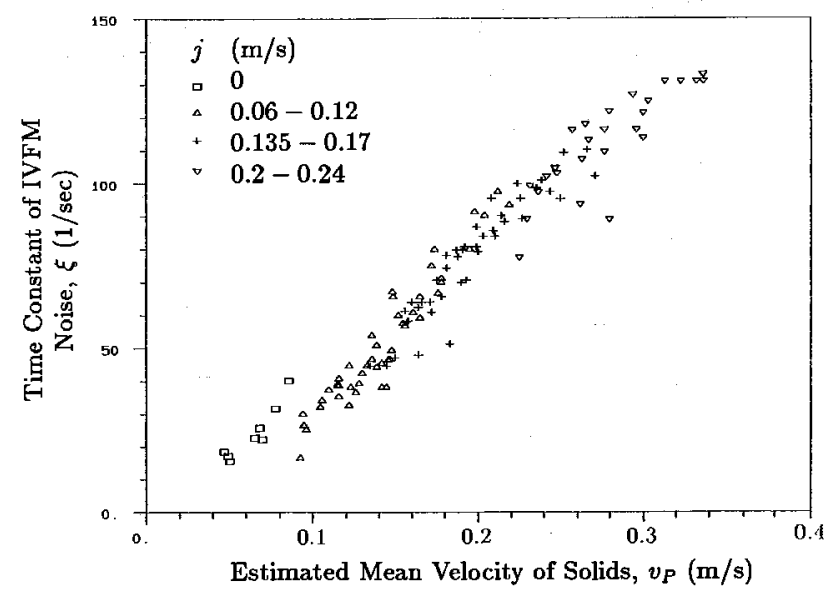

Fig. 14 Time constant $\xi$ of the fluctuating IVFM signal in solids-water flows versus an estimate of the average particle velocity based on the drift flux model 
disperse medium pressure caused by bubble-particle interactions. This effect is consistent with the enhanced erosive property of three component flows.

At low volume fractions ( $\alpha, \nu<17$ percent) the flows are dispersed and well behaved. The small scale of the structure is revealed in a time lapse photograph of such a flow in Fig. 15. However, a flow regime transition was observed above a certain combination of volume fraction values. Above this threshold, the homogeneous three-component distribution is observed to become unstable and is replaced by an unsteady flow regime with yortical structure; this is called the agitated vortical regime. Regime transition is observed to take place at solid and air volume fractions of approximately 17 percent. The vortices created have the same typical size as the pipe diameter (4 in.). This is seen in a time lapse photograph in Fig. 16. At high solid fractions the air flow is hardly visible, indicating that the bubbles tend to flow in the central region of the pipe. At larger air flow rates, groupings of air bubbles and slugs do however become visible, typically in the center of vortices. Thus, there exists a segregation effect of the vortices on the dispersed components. A flow regime map is presented in Fig. 17 showing the prevalence of dispersed flows at lower volume fractions of both bubbles and solids, and the onset of agitated flows at higher values.

6.2 Average Air Velocity in a Three-Component Flow. The average air velocity $v_{g}$ in the three-component flows studied was computed from the total air flux $j_{g}$ and the calculated air volume fraction $\alpha$.

$$
v_{g}=\frac{\dot{j}_{g}}{\alpha}
$$

We now introduce the average air velocity relative to the combined flux of liquid and solids, $v_{g(p)}$, which is defined by:

$$
v_{g(p l)}=v_{g}-j_{p}-j_{l} \text {. }
$$

The velocity $v_{g(p l)}$ indicates the extent of flow interference between the bubbles and the liquid-solid medium. This quantity is plotted in Fig. 18 against the total volume fraction of the

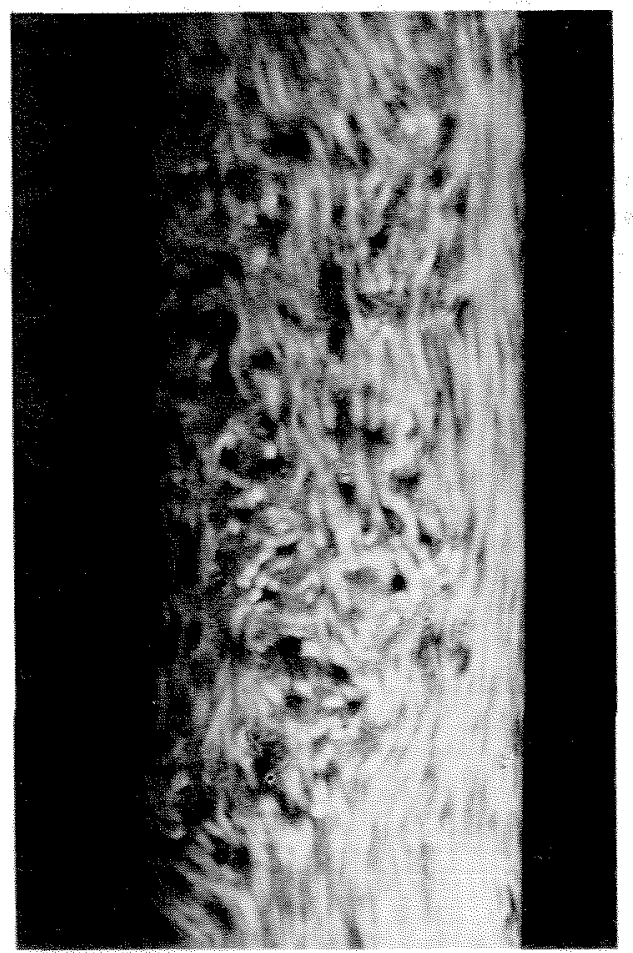

Fig. 15 Three component tlow of 12 percent solids volume fraction and 15 percent alr volume fraction, showing the small scale structure of disperse flow. $1 / 30$ second exposure was used. medium $(\alpha+p)$. It can be seen that flows which do not exhibit large vortical structure have small relative gas velocities, less than $.05 \mathrm{~m} / \mathrm{s}$ at total volume fractions between 15 and 30 percent. After transition, the relative velocity in the agitated flows rises sharply up to about $.35 \mathrm{~m} / \mathrm{s}$. This sudden increase in the relative velocity is characteristic of a change in regime as was shown by a number of investigators for air-water flows (these results are summarized by Wallis (1969) in his book). In their low Reynolds number work on suspensions, Fessas and Weiland (1981) observed similar behavior in the settling velocity of heavy particles in a bidisperse medium. They present the settling of velocity of one species as a function of the concentration of the other, with the concentration of the first as a parameter. The curves display the same initial decrease that we find followed by a sharp increase after a minimum value is reached. Visual observation of their sedimenting flows revealed segregation of species into vertical fingers subsequent to the regime change. This is in contrast to

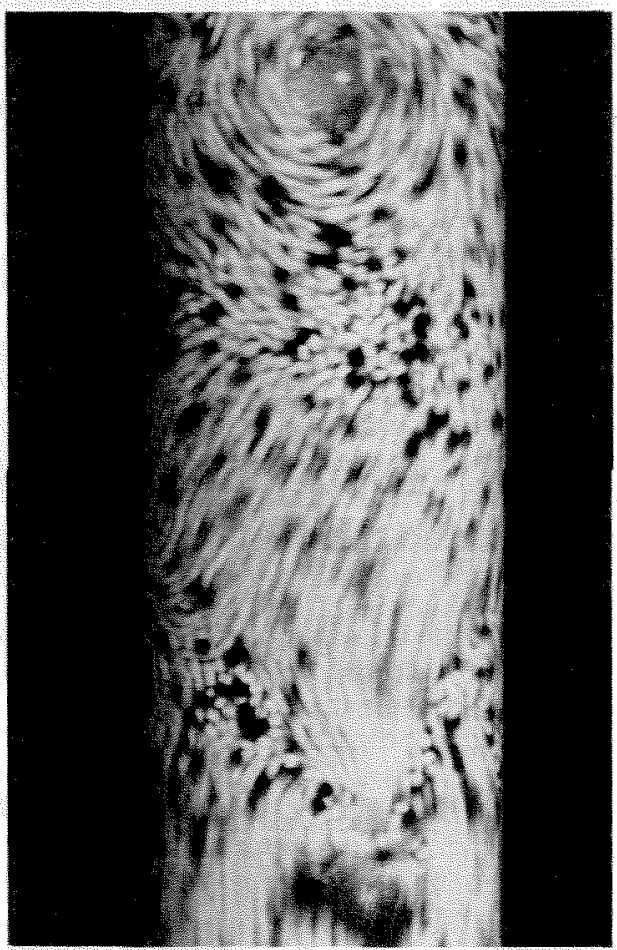

Fig. 16 Three component flow of 30 percent solids volume fraction and 15 percent air volume fraction, showing large vortex structure. 1/30 second exposure was used.

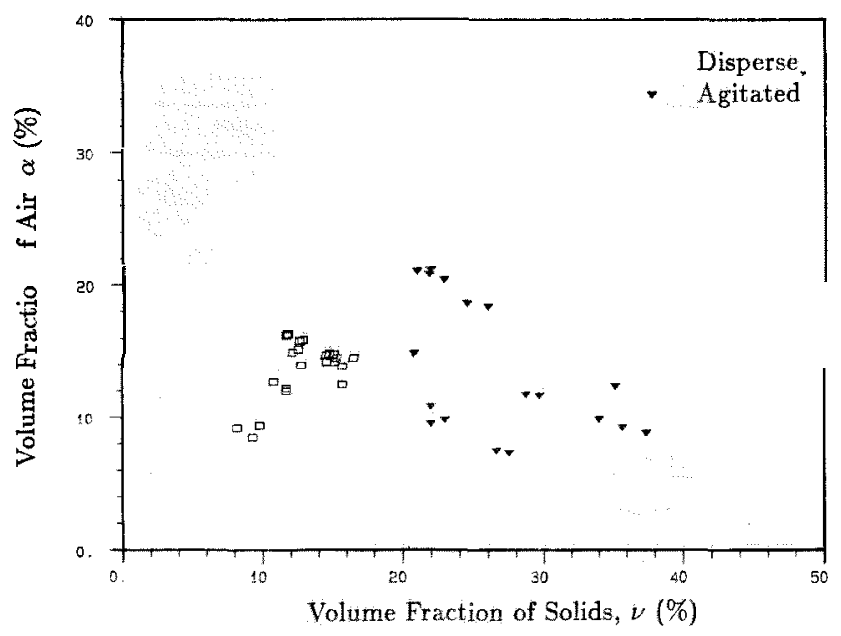

Fig. 17 Flow regime map of three component flows based on visual observation 


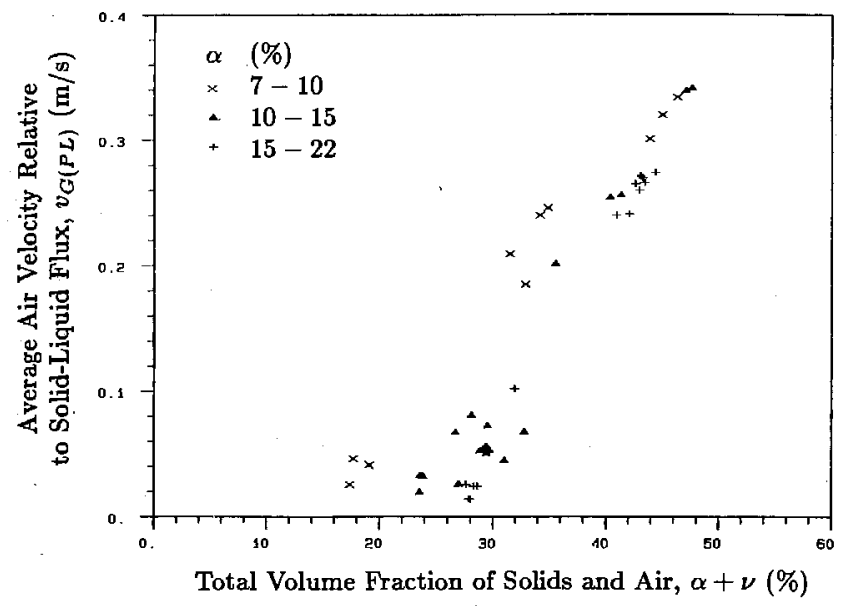

Fig. 18 Average velocity of air relative to the combined flux of liquid and sollds versus total volume fraction of disperse material

the present flows, which manifest large vortical structure subsequent to the loss of stability of the disperse regime.

All the relative velocity data falls on the same general curve when presented as a function of the total volume fraction of the disperse medium. This is also true for Fessas and Weiland's data though they do not make note of this fact. This property was assumed in a low Reynolds number bidisperse model by Lockett and Al-Habbooby (1974) and proved to give good agreement with experimental data. It therefore appears that this property holds not only for flows of small particle Reynolds number but also for the higher Reynolds numbers of about 1000 which pertain to this study.

6.3 Statistical Properties of Volume Fraction Signals in Three-Component Flows. We now turn attention to the statistical properties of the fluctuations in the measured volume fraction signals of three-component flows. In Sections 4 and 5 we showed that the amplitude of the fluctuations presented in the form of the Signal To Noise Ratio and the coherent time scale presented in the form of a time constant $\xi$ both contained valuable information on the nature of twocomponent flows.

In the three-component flows, the STNR shown in Fig. 19 displays a nearly linear dependence on total volume fraction, with a scatter of 5 percent. The STNR of well behaved disperse flows coincide with both bubbly and particle disperse flow values shown on Figs. 8 and 13. The flows which exhibit large vortical structure assume STNR values below the disperse flow curves. The small scatter in the results indicates that this type of measurement has potential as a means of monitoring the total volume fraction. The STNR exhibits the largest deviation from the disperse flow curves for flows of very low solids volume fraction resembling churn-turbulent flows. This is exemplified by the STNR curve for air-water flows, Fig. 8.These are the conditions under which the STNR would be least accurate as a measure of total volume fraction.

The coherent time scale of the noise in the three-component flows is presented in the form of the time constant $\xi$ versus the average air velocity in Fig. 20 for a wide range of gas volume fractions. Surprisingly, all points fall on one common curve with a maximum which coincides with the onset of vortical motion in the flow. The maximum value of $\xi$ is 75 with a corresponding average gas velocity of $.14 \mathrm{~m} / \mathrm{s}$. All well behaved dispersed flows fall to the left of the maximum and all agitated flows to the right. Note that the behavior of the time constant in churn-turbulent two-component flows (Fig. 9) was markedly different. The right-hand part of the curve which shows a

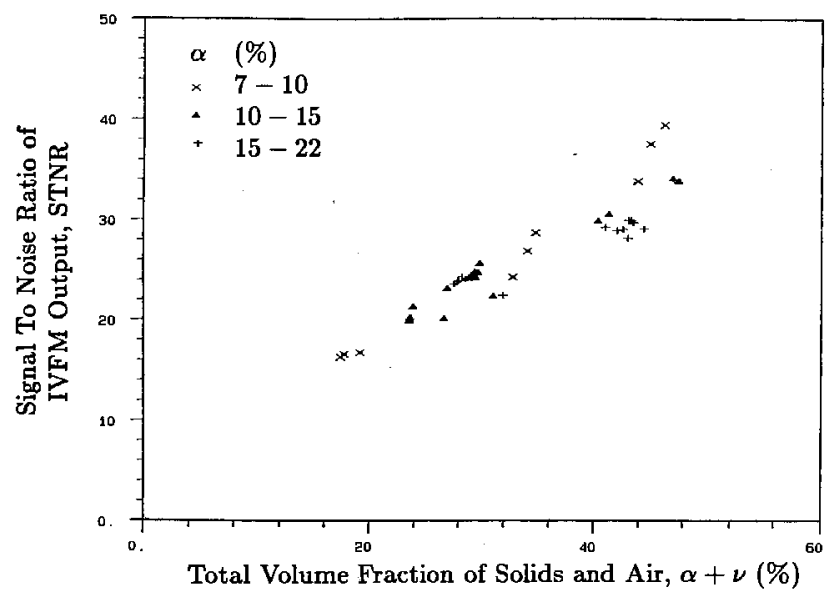

Fig. 19 Signal to noise ratio of the IVFM output in three component flows versus total disperse material volume fraction

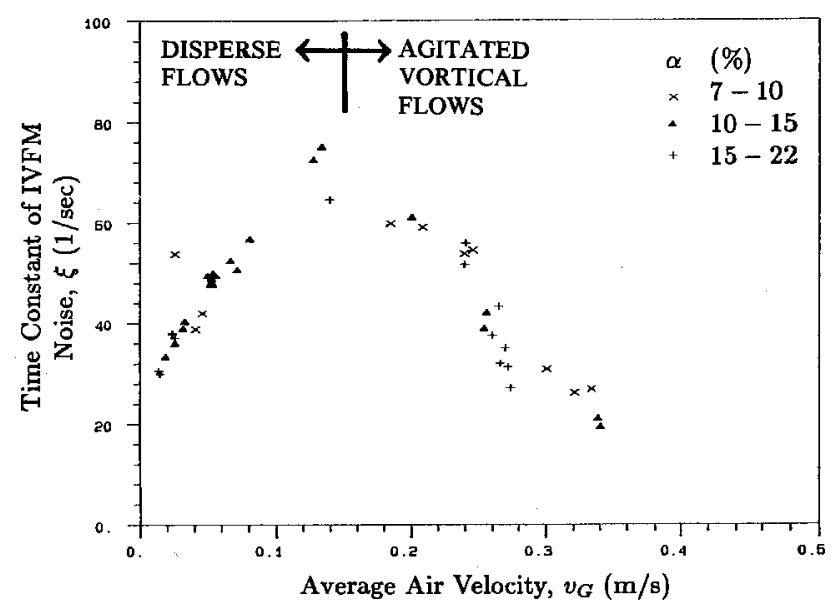

Fig. 20 Time constant $\xi$ of IVFM output fluctuations in three component flows versus the average air velocity

decreasing time constant implies the development of large scale vortical structure in the flow.

\section{Conclusion}

In this study, the nature of vertical two- and threecomponent gravity driven flows was studied. Gas-liquid and gas-liquid-solid mixtures were found to undergo a transition from a disperse to an agitated regime. The agitated vortical air-water-solids flows were so named for their distinct eddies of the size of the pipe diameter.

Solid-liquid flows, however, were found to remain quite homogeneously distributed for all volume fractions and flowrates considered. In agitated vortical three-component flows, segregation of species was observed. Air bubbles formed groupings in the center of large vortices, and slugs were occasionally created. This regime exhibited a dramatic increase in the gas flux relative to the solid-liquid mixture. A map of three-component flow regimes is presented in terms of the volume fractions of the individual constituents showing the regions of disperse and agitated vortical flows.

The structure of these flows was quantitatively analyzed by making use of the statistics in the fluctuations of volume fraction measurements. The statistical properties of the fluctuations were presented in terms of two properties of their autocorrelation function, namely its peak value (at $\tau=0)$ which represents the fluctuations amplitude and its "fall off" time which is a measure of the characteristic coherent time scale in the "noise." The former is presented in the form of a Signal To Noise Ratio (STNR), and the latter as a time constant of 
coherence, $\xi$. In disperse bubbly and particle flows, the STNR was found to be directly related to the volume fraction of air or solids. Remarkably, the results for bubbles and particles were found to be virtually identical. This consistency of the measurement and the independence of the dimensionless STNR on the IVFM sensitivity (gain) suggests that the STNR may be a useful measure of volume fraction in locations of adverse conditions where recalibration of the IVFM is inconvenient if not impossible. The time constant, $\xi$, was found to be linearly proportional to the mean particle and bubble velocities in disperse two-component flow conditions. Furthermore, the constant of proportionality was found to be the same for both types of flow. On the basis of this result, $\xi$ could be used as a nonintrusive measure of the mean velocity of the flowing particulate or bubbly material. As the gasliquid and gas-liquid-solid flows develop large scale structure and become churn-turbulent and vortical respectively, both the STNR and $\xi$ are found to decrease.

The size and propagation speed of large scale structure in churn-turbulent and unsteady three-component flows remains unexplored. Future efforts should be concentrated on applying a combination of auto-correlation and cross-correlation measuring techniques using more than one transducer to investigate the size, speed and persistence of the observed structures.

\section{References}

1 Batchelor, G. K., and Janse Van Rensbury, R. W., 1986, "Structure Formation in Bi-Disperse Sedimentation," J. Fluid Mech., Vol. 166, pp. 379-407.

2 Bernier, R. N., 1981, "Unsteady Two-Phase Flow Instrumentation and Measurement," Ph.D. thesis, California Institute of Technology.

3 Fessas, Y. P., and Weiland, R. H., 1981, "Convective Solids Settling Induced by a Buoyant Phase," AIChE Journal, Vol. 27, No. 4.

4 Ishagai, S., Yamane, M., and Roko, K., 1965, "Measurement of the Component Flows in a Vertical Two-Phase Flow by Making Use of the Pressure Fluctuations," Bull. JSME, Vol. 8, pp. 375-390.

5 Jones, O. C., and Zuber, N., 1975, "The Interrelation between Void Fraction Fluctuations and Flow Pattern in Two-Phase Flow," Int. J. Multiphase Flow, Vol. 2, pp. 273-306.

6 Kytömaa, H. K., 1986, "Stability of the Structure in Multicomponent Flows," Ph.D. thesis, California Institute of Technology.

7 Lockett, M. J., and Al-Habbooby, H. M., 1974, "Relative Particle Velocities in Two-Species Settling," Power Technol., Vol. 10, pp. 67-71.

8 Wallis, G. B., 1961, One Dimensional Two-Phase Flow, McGraw-Hill, New York.

\title{
Call for Papers
}

\author{
Symposium on Scaling of Fluids Engineering Models \\ 1989 ASME Fluids Engineering Division Spring Conference \\ University of California at San Diego, La Jolla, California \\ July 9-12, 1989
}

The Fluid Mechanics Committee, the Fluid Transients Committee, and the Coordinating Group for Fluid Measurements of the ASME Fluids Engineering Division are jointly organizing a Symposium on Scaling of Fluids Engineering Models for the ASME Fluids Engineering Spring Conference in July, 1989.

\section{SCOPE}

The objective of this symposium is to provide a technical forum for the presentation and discussion of the recent advances in the area of fluids engineering modeling. Typical topics include, but are not limited to:

- Scaling of Closed Conduit Flow Models

- Scaling of Free-Surface Flow Models

- Scaling of Fluid Machinery Models

- Scaling of Unsteady Flow and Transient Flow Models

- Scaling of Atmospheric Dispersion and Wind Engineering Models

- Scaling of Multiphase Flow Models

- Scaling of Movable Bed Models

- Scale Effects in Model Tests

- Extrapolation of Model Data

\section{SELECTION OF PAPERS}

Prospective authors should submit a 500 word abstract by July 1, 1988. Authors will be notified of acceptance by August 1, 1988. Draft copies of the full papers must be submitted by October 1, 1988 for formal review by the organizing committee. Authors of accepted papers will be notified by January 15, 1989 and receive a set of mats for typing the final paper draft. Papers are limited to a maximum length of six mat pages, including figures, and must conform to the editorial standards for FED publications. The final paper draft on mats must be submitted by March 1, 1989. Accepted papers will be published in a bound volume available at the Symposium.

\section{ORGANIZERS}

Abstracts may be submitted to any of the organizing committee members:

Dr. M. Padmanabhan

Alden Research Laboratory

30 Shrewsbury Street

Holden, MA 01520

(617) 829-4323
Dr. T. B. Morrow

Southwest Research Institute

P.O. Drawer 28510

San Antonio, TX 78284

(512)ntonio, TX 78284
Dr. S. Vigander

Tennessee Valley Authority Engineering Laboratory

P.O. Drawer E

Norris, TN 37828

(615) 632-1946 